Original Research Article

\title{
Interaction of Cetylpyridinium Chloride and Methylene Blue in Methanol-Water Solvent Media
}

Lalita Shrestha ${ }^{l}$, Debendra Rail, Prince Subba ${ }^{2}$ and Ajaya Bhattarai ${ }^{3}$ *

'Department of Chemistry, Central Campus of Technology, Dharan, Tribhuwan University

${ }^{2}$ Department of Microbiology, Central Campus of Technology, Dharan, Tribhuwan University

${ }^{3}$ Department of Chemistry, Mahendra Morang Adarsha Multiple Campus, Biratnagar, Tribhuwan

University

*Corresponding Author: Ajaya Bhattarai, Department of Chemistry, Mahendra Morang Adarsha Multiple Campus, Tribhuvan University, Biratnagar. E-mail: bkajaya@yahoo.com

\begin{abstract}
:
The interaction of Methylene blue with Cetylpyridinium chloride (CPC) in a series of solvents containing $0.1,0.2$ and 0.3 volume fractions of methanol in water were studied at room temp $(300 \pm 2 \mathrm{~K})$ by UV-Vis spectrophotometer. The CPC concentrations were varied from $0.2 \times 10^{-3}$ to $2 \times 10^{-3} \mathrm{~mol} \mathrm{dm}^{-3}$. A fixed dye concentration of $2.5 \times 10-5 \mathrm{~mol}$ $\mathrm{dm}^{-3}$ was used for this purpose. The spectrum analysis was performed for both the dye solution and mixtures containing dye and surfactant of different concentrations. The dye solution showed the absorbance maximum at 664 $\mathrm{nm}$ and a shoulder at $610 \mathrm{~nm}$. In 0.3 volume fraction of methanol as solvent, the dye and surfactant mixed solution showed a shift in peak values towards shorter wavelength (blue shift) from peak of the dye solution. The shift in peak increased with increase in surfactant concentration up to $0.6 \times 10^{-3} \mathrm{~mol} \mathrm{dm}^{-3}$, beyond which no further shift increment occurred. In the case of 0.1 and 0.2 volume fractions of methanol in water, no shift in peak was found at all concentrations of dye and surfactant. The absorbance of dye and surfactant mixed solution decreased gradually in 0.2 volume fraction of methanol with increase in surfactant concentration, whereas no significant change in absorbance was seen in 0.1 volume fraction of methanol. The shift decreased gradually with decrease in methanol concentration in the solvent.
\end{abstract}

Keywords: Methylene Blue, Cetylpyridium chloride, Interaction, Absorbance, Methanol-water

\section{Introduction}

Surfactants are also known as surface active agents as they have property of lowering the interfacial tension between water and oily substance. Surfactants consist of hydrophilic part (water loving) head group and hydrophobic (water repelling) tail group that is why a surfactant is amphiphilic molecule (Niraula et al., 2017). Cationic surfactants are used as cationic softeners, lubricants, retarding agents and antistatic agents and are more useful than other classes of surfactants (Bhattarai et al., 2017). Cetylpyridinium chloride is a cationic quaternary ammonium surfactant which is effective in preventing dental plaque and reducing gingivitis (Asadoorian and Williams, 2008). Methylene blue is heterocyclic aromatic dye which is solid, odorless and dark green powder at room temperature.

Numerous works on interaction between dye and surfactant have been carried out as it has tremendous potential for industrial applications particularly in textile industry, biochemistry, photochemistry, analytical chemistry and pharmaceutical purposes. Various types of interactions such as electrostatic interactions, hydrophobic interactions, hydrogen bonds, pi-stacking, cation-pi interactions, and van der Waals forces exist between dye molecules and surfactant aggregates (Karukstis et al., 2010). These types of interaction have been supported by most published data, e.g., dyes incorporated into micelles (Dutta et al., 2008), premicellar dye-surfactant complexes (Shah et al., 2008), ion pair aggregation (Dutta et al., 2008) and changes in the chromophore micro environment (Estelrich and Sabat, 2003).The strength of the molecular association depends on several factors including the dye concentration and structure, temperature, solvent and other factors. Researches have confirmed that surfactants affect the electronic absorption spectra of dye solutions. 
Different methods such as UV-Vis spectrophotometric (Akbas and Kartal, 2005), conductometric (Bracko and Span, 2000) and ion selective (Lopez-Fontan et al., 2006) have been extensively used to study the interaction between surfactant and dye. However, there has been little literature on work related to the effect of the solvent medium on the interaction between dyes and cationic surfactants. Therefore, this paper aims to study the effect of solvent composition (methanol- water mixture) on the interaction of MB and CPC by UV-Vis Spectroscopy.

\section{Materials and Methods}

\section{Preparation of methanol and Methanol-water solvent:}

Methanol (E. Merck, India) was distilled with phosphorous pentoxide, redistilled over calcium hydroxide and the collected fractions of methanol having specific conductance of 3-4 $\mu \mathrm{Scm}^{-1}$ at room temperature was used for solvent preparation. Deionized water (Stanbio Reagent (P) Ltd., Kolkata, India) was double distilled in the presence of potassium permanganate and the distilled water having specific conductance of 2-3 $\mu \mathrm{Scm}^{-1}$ at room temperature $(300 \pm 2 \mathrm{~K})$ was obtained. Methanol-water solvents containing $0.1,0.2$ and 0.3 volume fraction of methanol were prepared using redistilled methanol and water.

\section{Preparation of CPC Stock solution:}

CPC (Merck, Germany) was dried for $1 \mathrm{~h}$ in hot air oven at $373 \mathrm{~K}$ and $4.475 \mathrm{~g}$ of CPC was dissolved in $250 \mathrm{~mL}$ solvents containing $0.1,0.2$ and 0.3 volume fraction of methanol in water separately. After mixing, the sample solutions were kept overnight for equilibration.

\section{Preparation of Methylene blue Stock solution:}

Methylene blue (E. Merck, India) was dried for $1 \mathrm{~h}$ in hot air oven at $373 \mathrm{~K}$ and $0.0008 \mathrm{~g}$ of Methylene blue was dissolved in $100 \mathrm{~mL}$ solvents containing $0.1,0.2$ and 0.3 volume fraction of methanol in water separately. After mixing, the solutions were stirred with magnetic stirrer for about $4 \mathrm{~h}$ and kept overnight for equilibration.

\section{Spectrum analysis:}

Spectra were recorded by T $80+$ UV/VIS Spectrophotometer (PG Instruments Ltd., UK) at room temperature $(300 \pm 2 \mathrm{~K})$ using quartz cuvette of $1 \mathrm{~cm}$ (matched pair of $10 \mathrm{~mm}$ path length cuvette). UV-Vis spectra of dye solution at $2.5 \times 10^{-5} \mathrm{moldm}^{-3}$ in all volume fractions $(0.1,0.2$ and 0.3$)$ of methanol in water solvent media at room temperature were recorded. UV-Vis spectra of the dye and surfactant mixture were also recorded. The CPC concentration for this purpose was varied from $2.0 \times 10^{-3}$ to $0.2 \times 10^{-3} \mathrm{moldm}^{-3}$ for a fixed dye concentration of $2.5 \times 10^{-5} \mathrm{moldm}^{-3}$ in all volume fractions of methanol in water. All experiments were performed in triplicates at room temperature $(300 \pm 2 \mathrm{~K})$.

\section{Results and Discussion}

The electronic spectra of Methylene blue $\left(2.5 \times 10^{-5}\right.$ moldm $^{-3}$ ) in $0.1,0.2$ and 0.3 volume fraction of methanol in water showed a principal peak at about $664 \mathrm{~nm}$ and a shoulder roughly at $610 \mathrm{~nm}$. The peak in spectra of Methylene blue solution may be due to $n \rightarrow \pi^{*}$ transition of lone pair of electrons present in sulphur and nitrogen atoms of dye molecule. The electronic spectra of CPC in solvent containing 0.3 volume fraction of methanol showed absorption bands having peaks roughly at 250 and $215 \mathrm{~nm}$ and a valley at roughly $230 \mathrm{~nm}$ (Figure 1). Similar spectra were also observed for 0.2 and 0.1 volume fractions of methanol-water solvents. This result was similar to that reported by Mehta et al. (2008).

For solvent containing 0.3 volume fraction of methanol, the surfactant-dye mixture showed a shift in peak towards lower wavelength (blue shift) from the peak of dye solution alone. A blue shift of about $25 \mathrm{~nm}$ was observed in $0.2 \times 10^{-3} \mathrm{moldm}^{-3}$ concentration of $\mathrm{CPC}$ which further increased to around $65 \mathrm{~nm}$ at the surfactant concentration of $0.6 \times 10^{-3} \mathrm{moldm}^{-3}$. The blue shift could be due to the fact that surfactant molecule dissociates into cation $\left(\mathrm{C}_{21} \mathrm{H}_{23} \mathrm{~N}^{+}\right)$ which interacts with the lone pair of electrons present in the dye molecule. As a result $n \rightarrow \pi^{*}$ transitions in the dye become difficult and hence the high energy is required for transition which results in blue shifts/towards lower wavelength in spectra of surfactant-dye mixture solutions. As the concentration of CPC was increased from 0.6 to $1.8 \times 10^{-3} \mathrm{moldm}^{-3}$, a band appeared at $600 \mathrm{~nm}$ whose intensity decreased gradually on increasing the concentration of CPC (Figure 2). This trend in the spectrum of $\mathrm{CPC}$ was consistent with the results observed by Dutta et al. (2008). The results may be due to the formation of micelles of CPC which incorporates the dye molecules inside (Edbey et al., 2016) or the formation of surfactant aggregates with solubilized dye which resulted in the decrease in absorbance values of dye solution. 


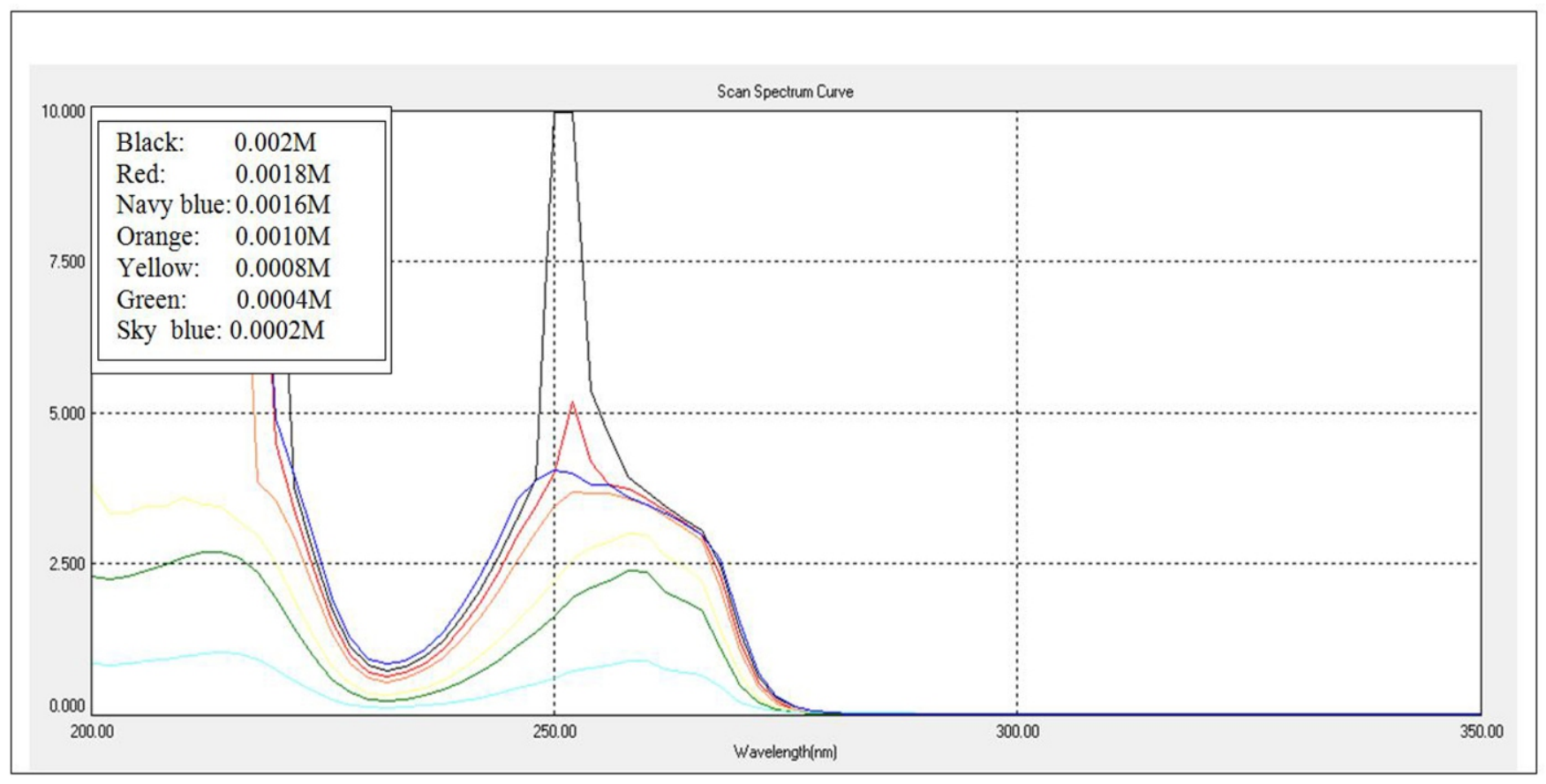

Fig 1. UV spectrum of $\mathrm{CPC}$ at different concentrations in the range $200-350 \mathrm{~nm}$ in 0.3 volume fraction of methanol at $300 \pm 2 \mathrm{~K}$

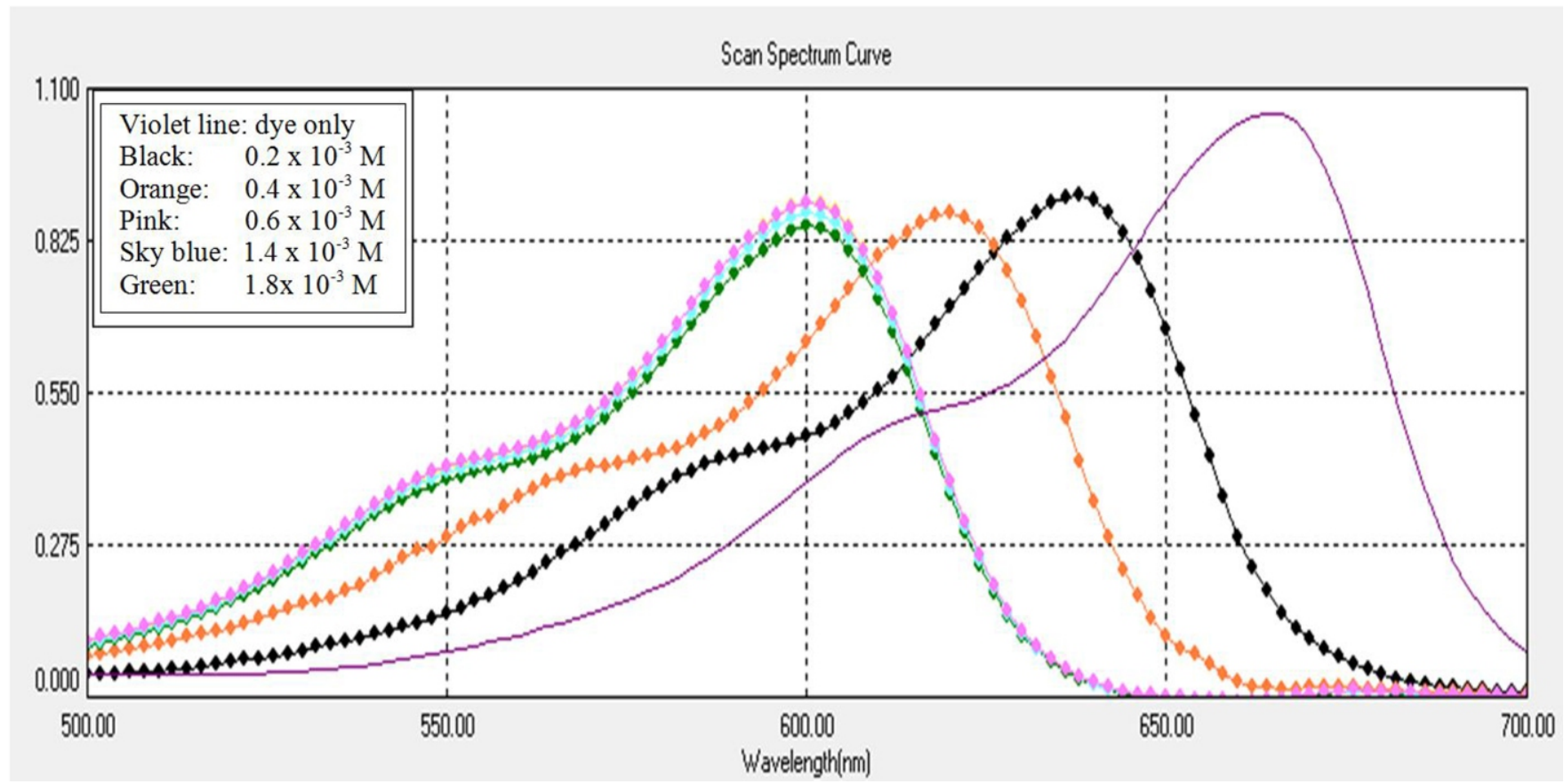

Fig 2. UV spectrum of dye $\left(2.5 \times 10^{-5} \mathrm{moldm}^{-3}\right)$ with various surfactant concentrations in 0.3 volume fraction of methanol in the range $500-700 \mathrm{~nm}$ at $300 \pm 2 \mathrm{~K}$. 


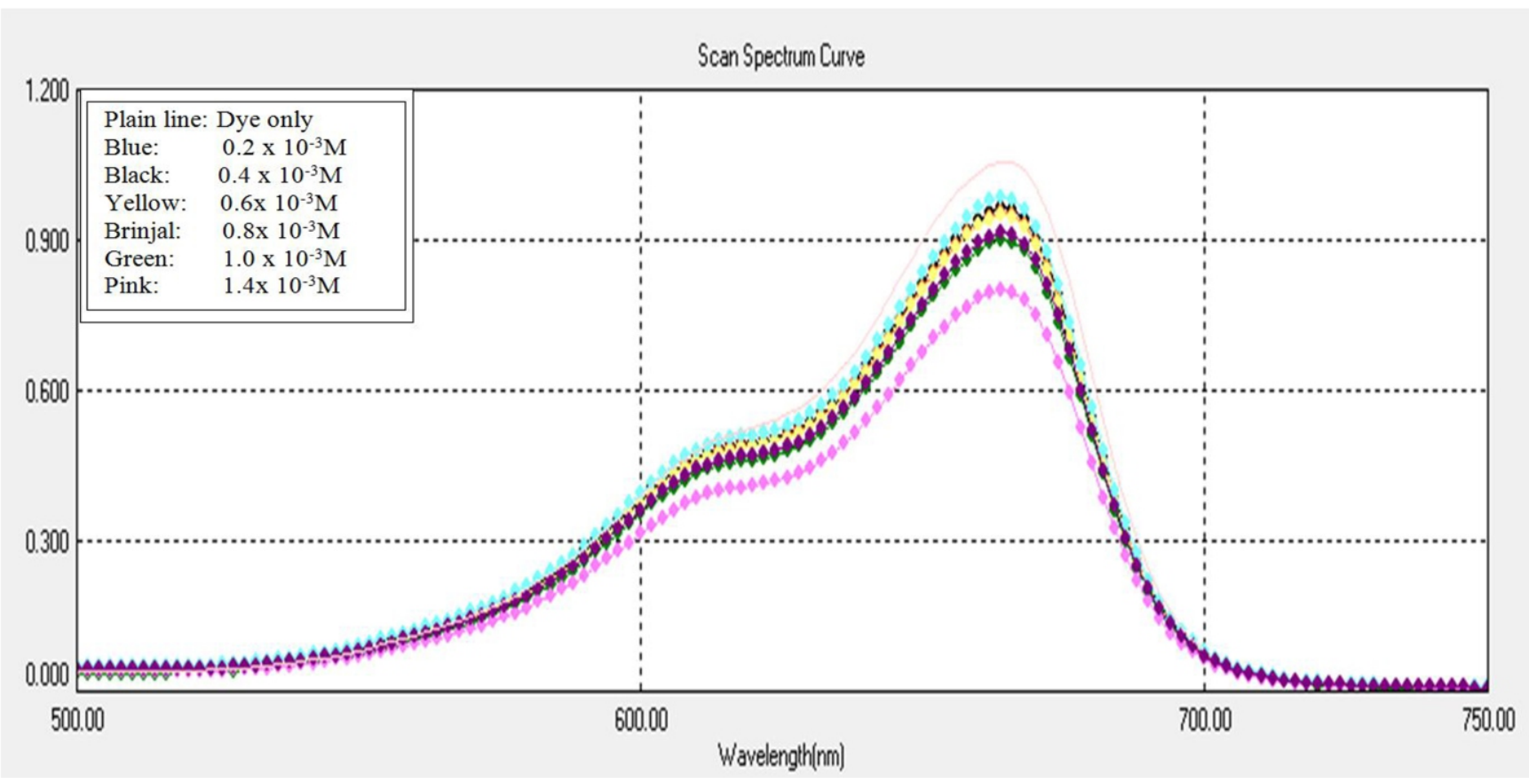

Fig 3. UV spectrum of dye $\left(2.5 \times 10^{-5} \mathrm{moldm}^{-3}\right)$ with various surfactant concentrations in 0.2 volume fraction of methanol in the range $500-750 \mathrm{~nm}$ at $300 \pm 2 \mathrm{~K}$.

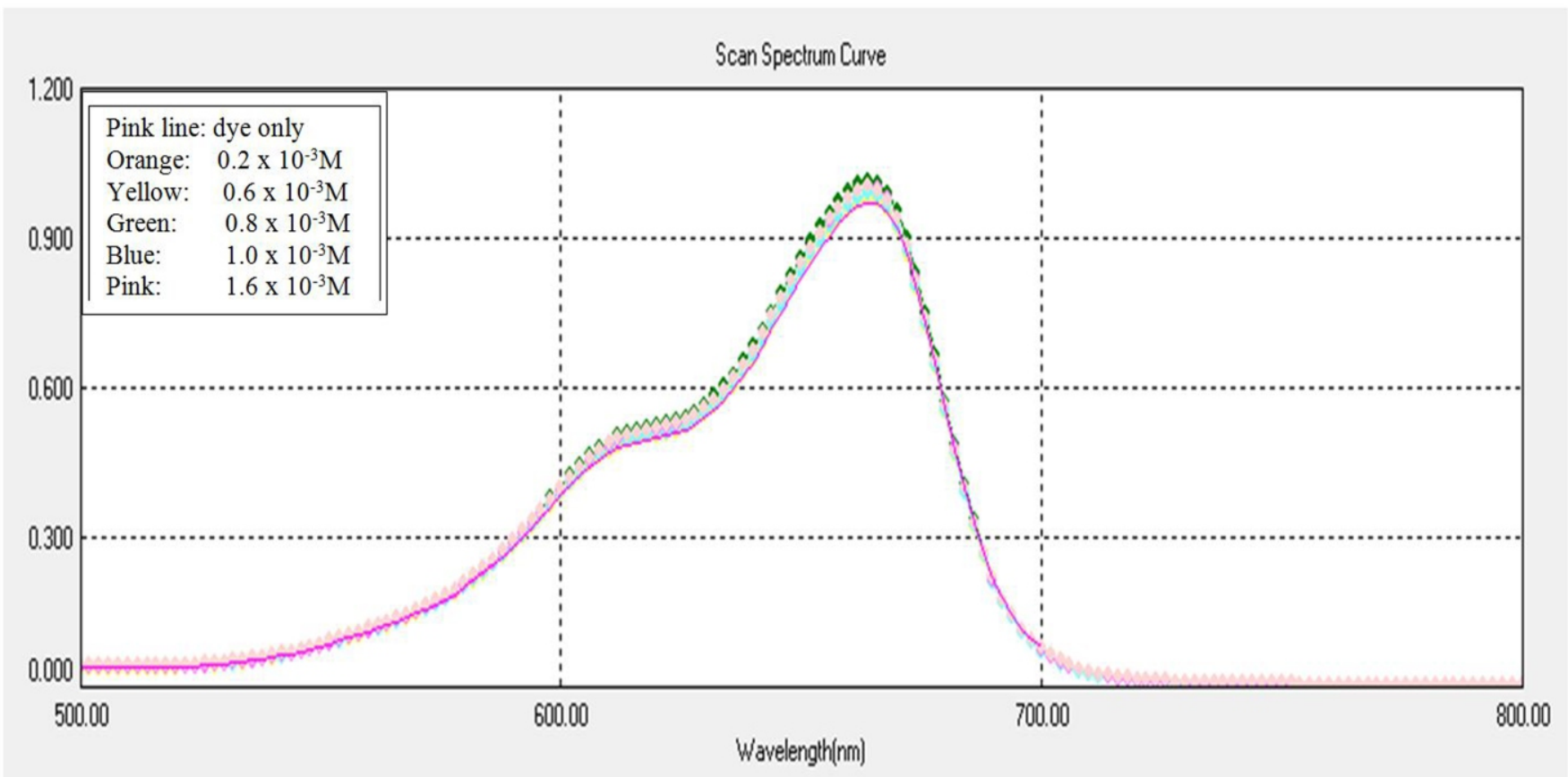

Fig 4. UV spectrum of dye $\left(2.5 \times 10^{-5} \mathrm{moldm}^{-3}\right)$ with various surfactant concentrations in 0.1 volume fraction of methanol in the range $500-800 \mathrm{~nm}$ at $300 \pm 2 \mathrm{~K}$. 
The absorbance of dye $\left(2.5 \times 10^{-5} \mathrm{moldm}^{-3}\right)$ with varying concentration of surfactant in solvent containing 0.2 volume fraction of methanol showed a peak at $664 \mathrm{~nm}$ and a shoulder at about $610 \mathrm{~nm}$ (Figure 3). As concentration of CPC was increased gradually, decrease in absorbance values was observed but the peak did not shift. No significant shift in peak and change in absorbance values were seen in mixture of dye-surfactant solution from peak of dye solution in solvent containing
0.1 volume fraction of methanol (Figure 4). The insignificant shift in the peak and very small change in the absorbance value indicated that there was only small or insignificant interaction between the surfactant and dye in 0.1 volume fraction of methanol. These results indicated that as the methanol fraction in the solvent was decreased, the interaction between CPC and Methylene blue also decreased.

\section{Conclusion}

There existed a significant interaction between MB and CPC in methanol-water solvent media. The interaction between dye and surfactant was the strongest in 0.3 , medium in 0.2 and weakest in 0.1 volume fraction of methanol.

\section{Acknowledgment}

The authors would like to acknowledge the Dean office, Institute of Science and Technology, Tribhuwan
University, Kathmandu, Nepal for granting the financial support to carry out this research project.

\section{References}

Akbas H and Kartal C. Spectrophotometric studies of anionic dye-cationic surfactant interactions in mixture of cationic and nonionic surfactants. Spectrochimacta A, Mol Biomolspectrosc, 2005, 61(5), 961-6.

Asadoorian J and Williams KB. Cetylpyridinium chloride mouth rinse on gingivitis and plaque. Journal of Dental Hygiene, 2008, 82 (5), 42.

Bhattarai A, Adhikari CN and Shrivastav GS. Micellization Behavior of Cetyl trimethyl ammonium bromide in Presence and $\mathrm{Absence} \mathrm{Na}_{2} \mathrm{SO}_{4}$ and $\mathrm{MgSO}_{4}$ in Aqueous media. Bibechana, 2017, 14, 77-85.

Bracko S and Span J. Conductometric investigation of dye-surfactant ion pair formation in aqueous solution. Dyes Pigments, 2000, 45, 97-102.

Dutta RK, Gohain B and Sarma S. Protonated dye-surfactant ion pair formation between neutral red and anionic surfactants in aqueous submicellar solutions. Journal of Molecular Liquids, 2008, 142, 130-5

Edbey K, Ttaib K, Benhmid A, Eltaboni F, Imragaa A and Alferjany A. Spectrophotometric and Conductometric Study of Methyl Orange- Cetylpyridinium Chloride Ion Pair in Aqueous Solution. British Journal of Applied Science \& Technology, 2016, 13(6), 1-5

Estelrich J and Sabat R. Determination of Micellar Microenvironment of Pinacyanol by Visible
Spectroscopy. Journal of Physical Chemistry B, 2003, 107, 4137-42.

Karukstis KK, Litz JP, Garber MB, Angell LM and Korir GK. A spectral approach to determine location and orientation of azo dyes within surfactant aggregates. Spectrochimica Acta Part A, 2010, 75, 1354-61.

Lopez-Fontan JL, Gonzalez-Perez A, Costa J and Sarmiento, F. The critical micelle concentration of tetraethyl ammonium perfluorooctylsulfonate in water. Journal of Colloid and interface science, 2006, 294(2), 458-65.

Mehta SK, Chaudhary S and Bhasin KK. Self-assembly of Cetylpyridinium chloride in water-DMF binary mixtures: A spectroscopic and physicochemical approach. Journal of Colloid and Interface Science, 2008, 321(2), 426-33.

Niraula TP, Chatterjee SK and Bhattarai A. Effect of Sodium Chloride on micellization of Sodium Dodecyl sulphate on water and methanol-water systems. Himalayan Journal of Science and Technology, 2017, 1, 61-4.

Shah SW, Naeem K, Naseem B and Shah SS. Complex formation study of hemicyanine dyes with sodium dodecyl sulfate by differential spectroscopy. Colloids and Surfaces A: Physicochemical and Engineering Aspects, 2008, 331, 227-31. 